\title{
Using intelligent technologies for knowledge formation in research on the impact of power industry on ecology and quality of life
}

\author{
Tatyana Vorozhtsova ${ }^{1, *}$, Dmitry Pesterev ${ }^{1}$, and Gleb $\mathrm{An}^{1}$ \\ ${ }^{1}$ MESI, Department of Intelligent Power Systems in the Energy Sector, Irkutsk, Russia
}

\begin{abstract}
The article discusses the possibilities of using intelligent technologies, namely, ontological and cognitive modeling to represent knowledge in studies of the impact of energy facilities on the environment and the quality of life of the population. The relevance of this work is due to the need to improve research methods. An intelligent information system and an ontological space of knowledge are being developed, integrating tools and an information base to carry out research. It is proposed to use ontologies to identify and organize the basic concepts of different subject areas related to joint research, establish relationships between them, as well as for the structural representation of knowledge. The cognitive modeling methodology is designed to analyze and model situations and make coordinated decisions in the energy industry, taking into account its impact on the environment and quality of life. Cognitive modeling is used to identify causal relationships between concepts, visualize them, describe possible situations and support to decision-making.
\end{abstract}

\section{Introduction}

The Melentiev Energy Systems Institute of Siberian Branch of the Russian Academy of Sciences (MESI SB RAS) is implementing the project "Methods for constructing an ontological space of knowledge for intellectual support of decision making in energy and ecology, taking into account the quality of life", supported by the RFBR grant No. 20-07-00195. This project envisages the development of an intelligent information system (IIS) that integrates a complex information base for carrying out research, mathematical and semantic methods, tools for assessing the impact of energy on the environment and quality of life. The presence and operation of energy facilities on the territory affects the quality of life of the population, since, on the one hand, it provides the needs for the necessary heat and electric energy, and on the other hand, it is one of the most serious sources of environmental pollution. The relevance of this work is due to the need to support for making agreed decisions in the field of energy research, taking into account its impact on the environment and quality of life. For this purpose, such methods of semantic technologies as, cognitive, and ontological modeling are developed and used. A unified ontological space of knowledge is being developed, which ensures the integration of research in the subject areas of energy and ecology. The developed system of ontologies provides information support for research by integrating the necessary data, designing and developing databases based on the ontological description. The use of ontological and cognitive modeling methods and supporting tools is proposed to assess the impact of energy facilities on the environment. Thus, the formation of an ontological knowledge space for research involves the integration and coordination of all necessary data, information and knowledge for the scientific justification of decisionmaking in the energy sector, taking into account its impact on the environment and quality of life.

\section{Opportunities and goals of ontological modeling}

For intellectual support of data from interdisciplinary research, taking into account the intersection of knowledge from different subject areas, it is proposed to use semantic technologies for describing and modeling knowledge. Domain knowledge modeling is the mainstream and basic paradigm of artificial intelligence. Ontological modeling is one of the leading areas of semantic modeling $[1,2]$. Currently, ontologies are the main approach to the development and implementation of knowledge management systems [3 - 5]. The application of ontological modeling in energy research was considered in the works of Massel L. V., Vorozhtsova T. N., Skripkin S. K., Kopaygorodsky A. $\mathrm{N}$. [6, 7]. At the same time, ontological engineering is used as the main method of working with knowledge and a fractal approach is used to knowledge structuring.

Ontological engineering is the process of designing and developing ontologies based on the structural analysis of the subject area [8].

Knowledge about a subject area is a collection of information about the objects of this subject area, the properties of these objects, the relationships between objects, as well as about the processes and situations that 
occur in this subject area. An ontology is usually constructed as a tree or network consisting of concepts and relationships between them. The ontological engineering process includes the following steps:

- identifying the basic concepts of a given subject area - concepts.

- identifying connections or relationships between concepts

- building a hierarchy of concepts

- description of the properties of the selected concepts.

The main advantage of ontological engineering is:

- systematic - ontology represents a holistic view of the subject area;

- uniformity - material presented in a uniform form is much better perceived and reproduced;

- scientific nature - the construction of an ontology allows you to restore the missing logical connections in their entirety.

The possibilities of applying the fractal approach to structuring knowledge and building an ontological space are described in the work of L. V. Massel [9].The fractal approach assumes that knowledge about the object under study is presented in the form of several layers, each of which characterizes a certain aspect of data, information or knowledge about the object.

Ontological models provide the following opportunities for working with knowledge:

- $\quad$ are a means of representing knowledge

- provide work with meaning of information

- allows automated processing

- provide application integration

- $\quad$ provide inference capability

In this work, the system of ontological models is designed for:

- coordination of research in different subject areas (energy, ecology, quality of life) in accordance with the project goals

- concordance of the concepts of these subject areas

- ensuring the availability and perception of large volumes of complex structured information

- descriptions of the structure and components of the AIS

\section{Components of the ontological knowledge space}

The system of ontologies of the developed ontological knowledge space for research includes knowledge representation for interrelated studies of energy, ecology, and quality of life.

The ontological model is based on a fractal approach, which presupposes the inclusion of several layers - meta-levels and their further stratification, which provides for an increasing degree of detail at each next level.

Metaontologies include basic concepts of the subject areas of energy, ecology and quality of life that are relevant to collaborative research. They are used as a basis for describing the components and structure of an intelligent information system, as well as for developing the system interface. The following levels of ontologies include:

- Ontological description of research sections

- Ontologies that describe the data and information used, database ontologies

- An ontological description of knowledge bases that contain descriptions of classes and instances, their properties and relationships, and a set of rules that allow logical inference.

Figure 1 shows metaontology, which combines the basic concepts of subject areas and shows the main research areas - energy, ecology, and quality of life. The presented concepts are the base classes of the ontology system. IIS components correspond to these sections.

The section "Power industry" contains a description of energy objects and their properties, on which the anthropogenic influence on the environment and the quality of life depends. The section "Ecology" describes anthropogenic factors, elements of the natural environment, and methods for assessing anthropogenic impact.

The concept of "Quality of life" is an aggregate characteristic of the living standard and objective and subjective living conditions of the population, which determine the physical, mental, socio-cultural development of a person, group or community of people [10]. According to the World Health Organization (WHO), it is the perception of individuals of their position in life in the context of the culture and value system in which they live, in accordance with goals, expectations, norms and needs. Accordingly, the section "Quality of life" includes a description of indicators of quality of life, factors of influence and methods for analyzing this influence.

Section "Task" contains a description of the tasks to be solved in these studies. Section "Methodology", as a component of IIS, includes a set of methodological approaches in research, methods and algorithms used to assess the impact of energy facilities on the environment and quality of life.

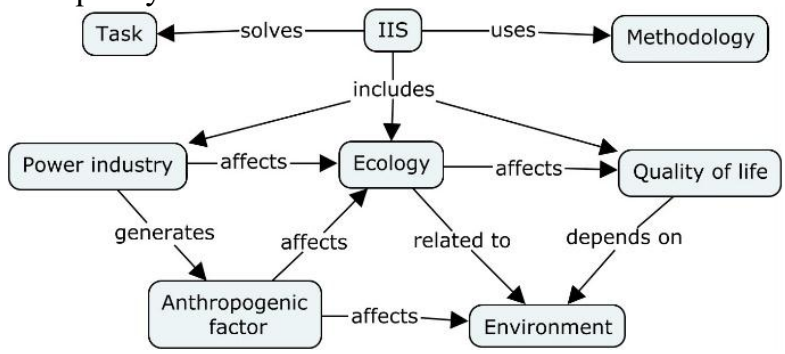

Fig. 1. Metaontology of knowledge of subject areas research

One of the important concepts of the presented metaontology is the "Anthropogenic factor" - the cause of anthropogenic impact on the environment, due to the process and conditions of the object's functioning, its characteristic features. In relation to energy facilities, anthropogenic factors include emissions, waste, radiation, noise, vibration, radiation, etc. The anthropogenic factor depends on the type and type of energy resource and the type of energy facility technologies. Using indicators of anthropogenic impact 
on the environment and elements of the environment, the degree of negative impact on the quality of life of the population is assessed at the expert level.

\section{Cognitive modeling and its application in these studies}

Cognitive analysis and modeling are modern intellectual technologies that are used to study weakly formalized and weakly structured systems, which include economic, social, and environmental systems [11]. Currently, the application of cognitive modeling is developing in the direction of modeling and analysis of situations and support to decision making. Abroad this is reflected in the works of Peter Grumpos and Chrysostomus Stylios [12, 13]. In our country, this is the work of employees of the Institute of management problems of the Russian Academy of Sciences [14, 15], the scientific school of Kulinich [16], etc. At MESI SB RAS it is proposed to use cognitive modeling as a tool for studying the problem of energy security (ES) $[17$, $18]$.

Cognitive analysis is based on the cognitive-target (cognitive) structuring of knowledge about an object and its external environment. Cognitive structuring is the identification of the most significant factors that affect the situation and the cause-and-effect relationships between them. The methodology of cognitive modeling is based on modeling the subjective views of experts about the situation. Experts knowledge is presented as a cognitive map.

In this paper, we propose to use cognitive modeling to analyze the mutual influence of energy and ecology factors on the quality of life of the population. The use of ontologies in this case is necessary to identify these factors and formulate concepts. In the ontological space, compiled for the studied interdisciplinary interaction, there are factors from one area, which can both positively and negatively influence factors from another. For example, factors from the field of energy have both a positive effect on the quality of life (use of electricity, use of thermal energy) and a negative one (environmental pollution, noise impact, etc.). Therefore, the main task of cognitive modeling is to determine the final influence of factors on each other in a particular situation. Another task is to visualize the dependencies between the main factors of the study area.

To build a cognitive map, the subject area is analyzed, and the main factors (concepts) that are important for research are identified. If there is already an ontology of the subject area, then concepts are allocated directly on the basis of this ontology. After this stage, connections and their nature are established between the concepts. In the simplest case, the relationships can be either positive or negative. In a more complex case, weight coefficients obtained by expert evaluation are added to the signs of connections. When analyzing a specific situation, the cognitive map is built either anew or selected from among existing ones, provided that it contains the necessary concepts of the subject area.

Figure 2 shows an example of a cognitive map that describes the main factors of the situation "providing the population with heat energy" from the position of the main factor "Quality of life".

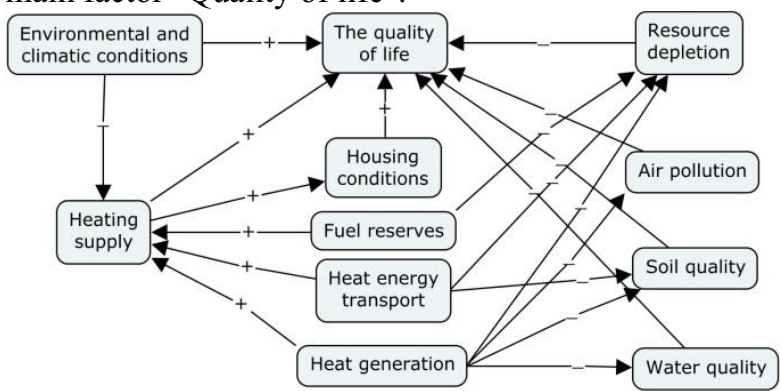

Fig. 2. Cognitive map "providing the population with heat energy".

This map shows the influence of factors on the "Quality of life" target factor. This influence is both positive and negative. To accurately determine the total impact, you will need to introduce weighting coefficients, which are determined by the method of expert assessments. In this case, it is possible to determine the final impact.

\section{Ontologies and data model}

Creation of information systems using the ontological approach provides a significant advantage in terms of the correct preparation of information components [19].

As noted, the developed system of domain ontologies for research involves several levels. Ontologies that describe the data and information used are the basis for modeling and developing databases, since the ontology is a convenient basis for developing a data schema [20]. One of the components of the developed intelligent information system is the database development module based on ontologies. As you know, a database consists of tables and data. An ontology is a connected graph from which you can get data, element values, and their generalizing properties. Generalizing properties of items should be interpreted as database tables, and values as data in tables. In Fig. 3, the concept of "Energy source" is presented as generalizing property of the objects "Diesel power station", "Thermal power plant", "Boiler house". In the database, we get the following structure: the table "Energy source" with the data "Diesel power station", "Thermal power station", "Boiler house".

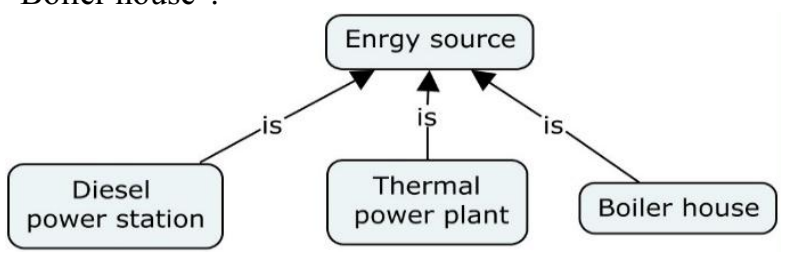

Fig. 3. Ontology "Energy source»

An example of such a table is shown in figure 4. 


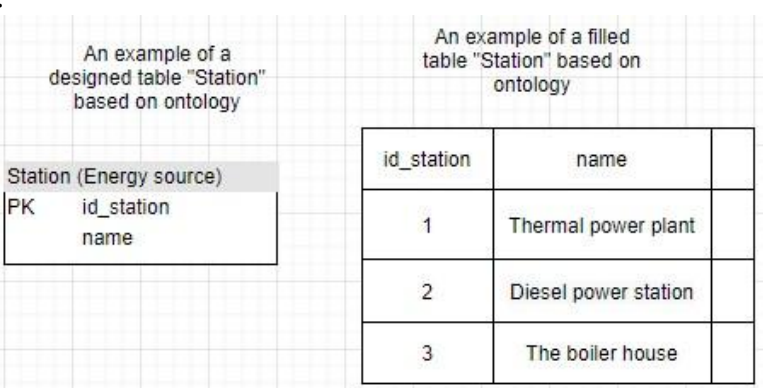

Fig. 4. Table "Energy source" of the designed database

The figure 5 shows a block diagram of the algorithm for converting an ontology into a database schema, and the figure shows a diagram of a database for storing information about an energy facility-a boiler house. Since the ontology uses natural language attributes, to align it with the database schema, it is necessary to limit the set of valid relationships between concepts ("has", "is", "measured in", "can take a value", "consists of").

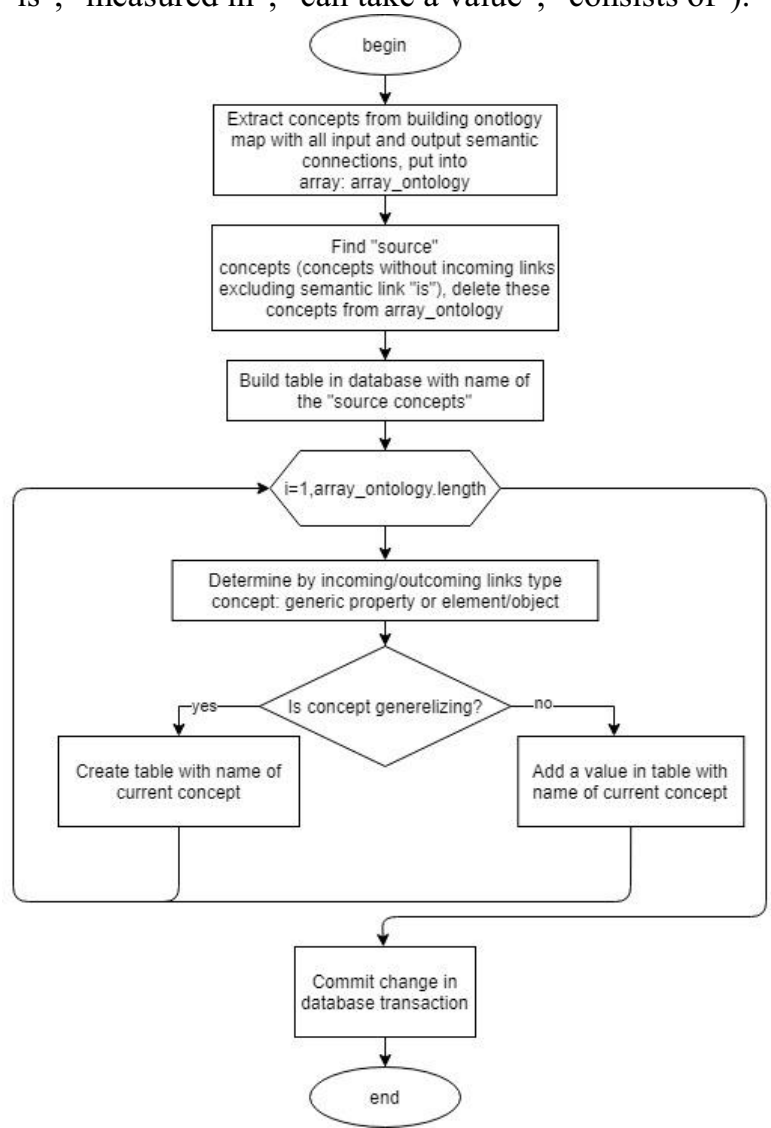

Fig. 5. Algorithm for converting an ontology into a database schema.

In the future, based on the constructed data model, an information system will be formed that will serve as information support for experts in supporting decisionmaking in the energy and environmental sectors.

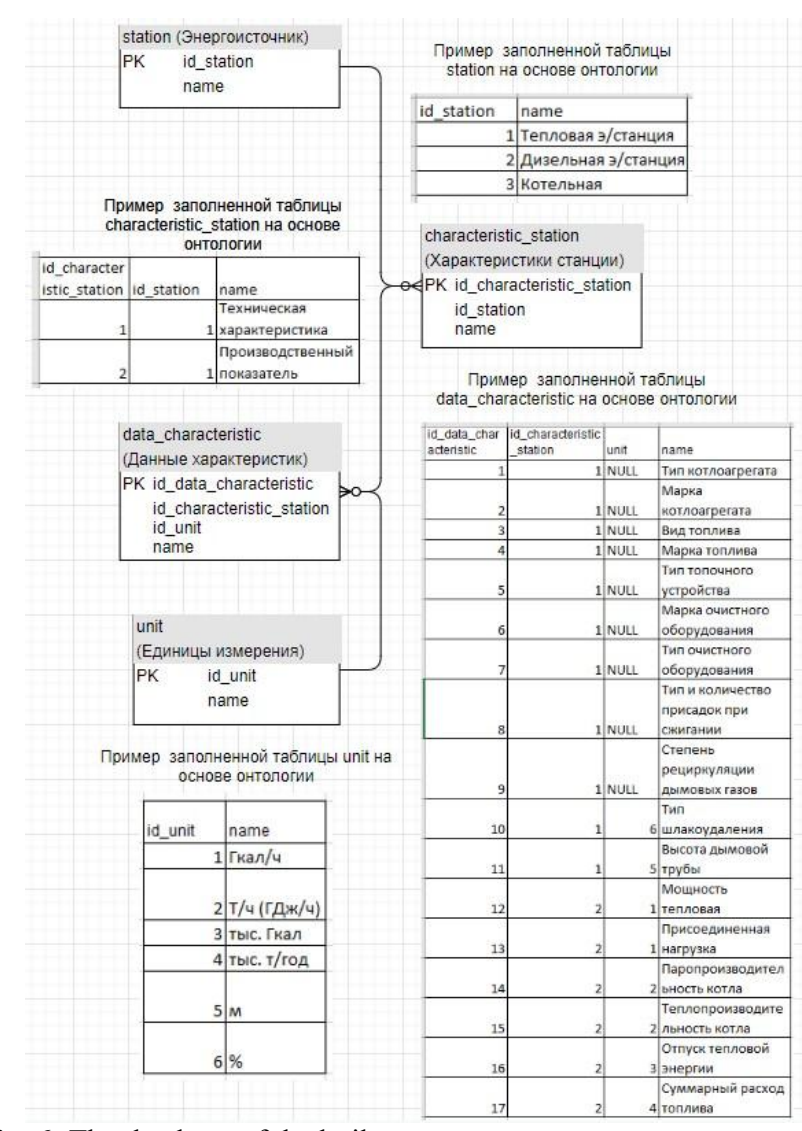

Fig. 6. The database of the boiler

\section{Conclusion}

The paper describes examples of using intelligent technologies to perform research on the impact of energy facilities on the environment and the quality of life of the population. For this purpose, an intelligent information system is being developed that integrates a complex information and methodological base of interdisciplinary research. Semantic modelling methods, including ontological and cognitive modelling), are used to support coordinated decision-making in the field of energy, taking into account its impact on the environment. A unified ontological knowledge space is being developed that provides integration of research in the subject areas of energy and ecology.

The developed system of ontologies provides information support for research by integrating the necessary data, designing and developing databases based on the ontological description. Ontologies are used to identify and organize the basic concepts of different subject areas that are relevant to joint research, establish relationships between them, as well as for the structural representation of knowledge.

Cognitive modeling is used to analyze and model situations and make coordinated decisions in the energy industry, taking into account its impact on the environment and quality of life. Cognitive models are used to identify cause-and-effect relationships between concepts, their visual representation in the process of describing possible situations and making decisions. 


\section{Acknowledgments}

The results were obtained within the framework of the project under the state assignment of the ISEM SB RAS AAAA-A17-117030310444-2 (project No. 349-20160005) and with partial financial support from RFBR grants № 19-07-00351, № 20-07-00195.

\section{References}

1. T.R. Gruber, Towards principles for the design of ontologies used for knowledge sharing // International Journal of Human-Computer Studies. V. 43. (5/6). 907 - 928. (1995)

2. N. Guarino, Formal Ontology and Information Systems // Proceedings of International Conference of Formal Ontology and Information Systems (FOIS'98), Amsterdam: IOS Press, P. 3 - 15. (1998)

3. A. Muñoz-García, K. Lagos-Ortiz, V. VergaraLozano, J. Salavarria-Melo, K. Real-Aviles, N. Vera-Lucio, Ontological Model of Knowledge Management for Research and Innovation. Technologies and Innovation 51-62 (2016)

4. L. Razmerita, A. Angehrn, A. Maedche,: Ontologybased user modeling for knowledge management systems. UM 2003. LNCS, vol. 2702. Springer, Heidelberg (2003).

5. Yu.A. Zagorulko, G.B. Zagorulko, Ontologies and their practical application in knowledge - based systems, All - Russian conference with international participation "Knowledge-Ontologies-Theories", Novosibirsk, Sobolev Institute of mathematics SB RAS, Volume 1, (2011)

6. L.V. Massel, T.N. Vorozhtsova, A.N. Kopaygorodsky, N.N. Makagonova, S.K. Skripkin, Application of ontologies in research and decision support in energy, All - Russian conference with international participation "Knowledge-Ontologies - Theories", Novosibirsk, SB RAS, Volume 2, 2938, (2013)

7. L.V. Massel, T.N. Vorozhtsova, N.I. Pyatkova, Ontological engineering to support strategic decision - making in the energy sector, Ontology of Design. . 7, №1 (23), 66-76, (2017)

8. T.A. Gavrilova, D.V. Kudryavtsev, D.I. Muromtsev Engineering of knowledge. Models and methods, St. Petersburg, 324, (2016)

9. L.V. Massel, Fractal approach to knowledge structuring and examples of its application, design Ontology, Vol. 6, №2 (20), 149-161, (2016)

10. Great Russian encyclopedia, https://bigenc.ru/vocabulary

11. R. Axelrod, Structure of Decision: The Cognitive Maps of Political Elite, Princeton University Press, 422, (1976)
12. P. Groumpos, C. Stylios, Modelling supervisory control systems using fuzzy cognitive maps, Chaos, Solitons \& Fractals, vol. 11(1-3), 329-336, (2000)

13. E. Papageorgiou, C. Stylios, P. Groumpos An integrated two-level hierarchical system for decision making in radiation therapy based on fuzzy cognitive map, IEEE Transactions on Biomedical Engineering, vol. 50(12), 1326-1339, (2003)

14. Z.K. Avdeeva, S.V. Kovriga, D.I. Makarenko. Cognitive modeling for solving problems of managing weakly structured systems (situations), № 16, 26-39, (2007)

15. Z.K. Avdeeva, G.B. Gorelova, S.V. Kovriga, Cognitive studies of the quality of life of the city population, Proceedings of the Seventh International forum on cognitive modelling, 205214, (2019)

16. A.A. Kulinich, Semiotic cognitive maps. Part 1. Cognitive and semiotic approaches in computer science and management, Management problem, № 1, 2-10, (2016)

17. A.G. Massel, Cognitive modeling in energy security research: applications and development prospects, Moscow, Fizmatlit, Vol. 2, 153-158, (2014)

18. L.V. Massel, Application of ontological, cognitive and event-based modeling to analyze the development and consequences of emergency situations in the energy Sector, Security and emergency issues, № 2, 34-43, (2010)

19. Ontological engineering and data model, https://studref.com/389454/informatika/ontologich eskiy_inzhiniring_model_dannyh

20. N.V. Golikov, Application of ontologies, http://www.ict.nsc.ru/ws/YM2006/10628/golikov. html 